\title{
Midline pyogenic granuloma of the tongue: A rare case report
}

\author{
P L Ravishankar ${ }^{1}$, Vandana Vijayan ${ }^{2 *}$, Sunanda Rao $^{3}$, A V Saravanan ${ }^{4}$ \\ ${ }^{1}$ Professor, ${ }^{2,3}$ Post Graduate, ${ }^{4}$ Reader, Dept. of Periodontics, SRM Katt. Dental College, Kanchipuram, Tamil Nadu, India
}

*Corresponding Author: Vandana Vijayan

Email: vandanaavijayan@gmail.com

\begin{abstract}
Pyogenic granuloma or granuloma pyogenicum of oral cavity is a benign non-neoplastic mucocutaneous lesion evolving in response to local irritation, its a fairly common lesion comprising approximately $1.8 \%$ of a large series of oral biopsies. A clinically and histologically similar lesion restricted to gingiva occurs in $0.5 \%$ to $2.7 \%$ of pregnant females and is called granuloma gravidarum or "pregnancy tumor". ${ }^{1,2}$ The unusual location of a pyogenic granuloma on midline of dorsum of the tongue in a pregnant patient prompted this case report.
\end{abstract}

Keywords: Midline, Pyogenic granuloma, Tongue.

\section{Introduction}

Pyogenic granuloma is a fast-growing reactive proliferation of endothelial cells that usually occurs in response to chronic irritation. ${ }^{3}$ It is a benign tumour of the soft tissue arising from the connective tissue of the skin or mucous membrane. The term is a contradiction as the lesion does not contain pus nor it is granulomatous. It was first described in 1897 by two French surgeons, Poncet and Dor. ${ }^{4}$ It is considered as a capillary haemangioma of lobular subtype as suggested by Mills, Cooper, and Fechner, which is the reason they are often quite prone to bleeding. The most common intraoral site is marginal gingiva, but lesions have been reported on palate, buccal mucosa, tongue, and lips. Extraoral sites commonly involve the skin of face, neck, upper and lower extremities, and mucous membrane of nose and eyelids. ${ }^{5,6}$

Being a non-neoplastic growth, excisional therapy is the best treatment of choice but some alternative procedures such as cryosurgery, excision by $\mathrm{Nd}$ :YAG Laser, flash lamp pulsed dye laser, injection of corticosteroid or ethanol, and sodium tetradecyl sulfate sclerotherapy have been reported to be effective. This is a case of a pyogenic granuloma of midline of dorsum of the tongue, a rare location of its occurrence. We present this case to enlighten the readers to keep pyogenic granuloma as a differential diagnosis while suspecting tumors of oral cavity especially in older patients.

\section{Case Report}

A 29-years old female in her sixth month of pregnancy visited the department of Periodontics, SRMKDC \& $\mathrm{H}$, Kancheepuram, Chennai complained of a painless mass on tongue. Her past medical history was unremarkable and general physical conditions were normal for women in sixth month of pregnancy. Oral examination revealed normal findings except for the tongue where a $6 * 6 \mathrm{~mm}$ erythematous, sessile, painless, rubbery to firm, dome shaped papule which is lobulated in appearance was found (Fig. 1). But often bled while eating, rinsing, or cleaning the tongue. The exact location of the papule was just right of midline in the anterior dorsal surface of her tongue. The mass had grown since more than 15 days. No lymph nodes were detected at palpation. A scalpel excision with $2 \mathrm{~mm}$ margins at the clinical boundary was performed under local anesthesia (Fig. 2). The lesion was also certainly dissected from the principal muscular layer. The patient experienced no adverse post-operative sequelae. The excised specimen was submitted for histological analysis.

\section{Histological Findings}

It showed parakeratinised stratified squamous epithelium of varying thickness with thin elongated rete ridges (Fig. 4). Dense fibrous connective tissue with fibroblasts, collagen bundles, endothelial lined blood capillaries, engorged RBCs, hemorrhagic areas, dense diffuse mixed inflammatory infiltrate consists of neutrophils, lymphocytes and plasma cells (Fig. 5). There was no mark of malignancy. The appearance was consistent with that of a pyogenic granuloma.

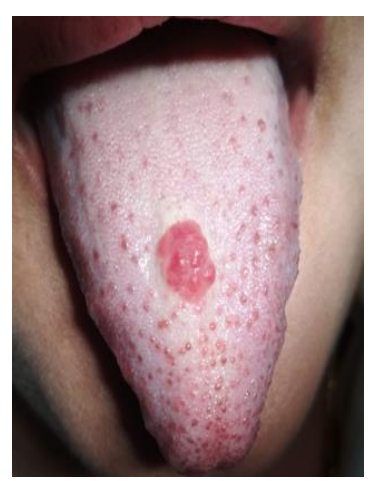

Fig. 1: Pre-operative

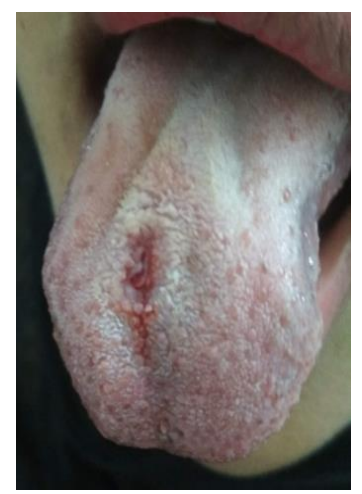

Fig. 2: Immediate post-operative 


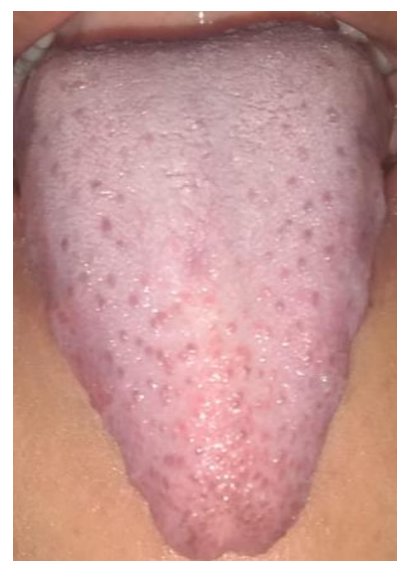

Fig. 3: Post operative (2 weeks)

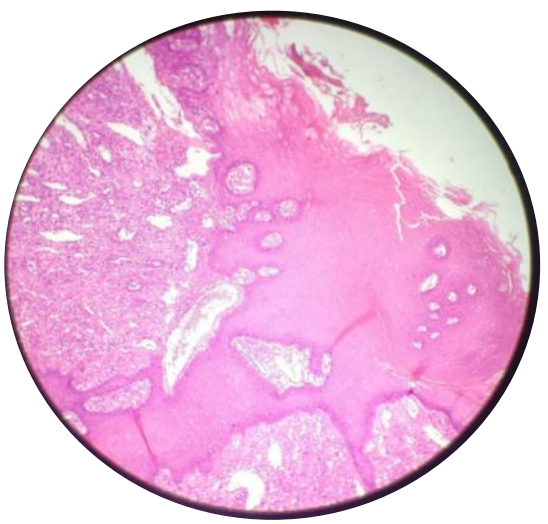

Fig. 4

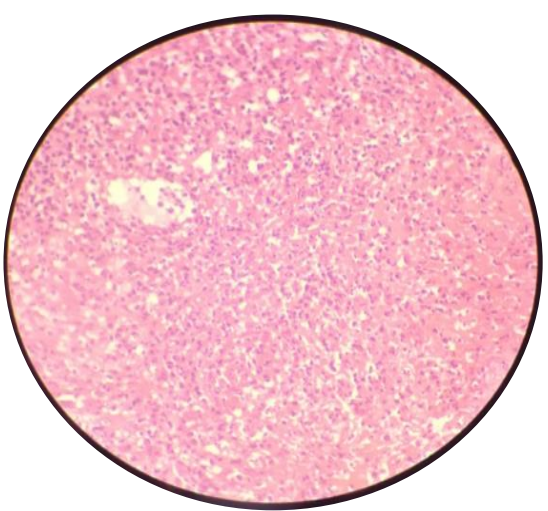

Fig. 5: Histopathology

\section{Discussion}

First described in humans by Poncet and Dor in 1897, pyogenic granuloma is a non-neoplastic inflammatory hyperplasia that usually occurs in response to chronic irritation. Given that these lesions are neither pus-producing nor granulomatous, the term pyogenic granuloma actually represents a double misnomer. Pyogenic granulomas can occur anywhere in the body and are common on the fingers and the toes around the nail beds. ${ }^{7,8}$ Within the oral cavity, pyogenic granulomas account for $1.85 \%-7 \%$ of all biopsy findings For Akyol et al. this rate ranges from 1.5 to $2 \%$. Although PG may occur in all ages, it is predominant in the second decade of life in young adult females, possibly because of the vascular effects of female hormones, especially during pregnancy. ${ }^{8}$ The clinical appearance is generally of a dull red, sessile, or pedunculated smooth surfaced nodule that may easily bleed, crust, or ulcerate. Lesions may grow rapidly, reach its maximum size, and remain fixed. They may typically begin as small, red papules that rapidly enlarge to become pedunculated raspberry-like nodules. Rarely, patient may develop multiple satellite angiomatous lesions after removal of a solitary pyogenic granuloma.

Oral pyogenic granulomas show a prominent tendency for the gingiva which accounts for $75 \%$ of all cases. Gingival irritation and inflammation that result from poor oral hygiene may be a triggering factor in many patients. The lip, tongue, and buccal mucosa are the next most common locations. Hormonal influences have been frequently suggested and the so-called pregnancy tumor is nothing more than a pyogenic granuloma that appears to arise more readily in the hormonally primed gingiva. Estrogens may increase vascular endothelial growth factor levels. The tumor usually reverts postparturition. ${ }^{9}$

"In most of the cases, minor trauma and or chronic irritation are mentioned in the etiopathogenesis of pyogenic granuloma. Infection may play a role with suggestions of agents such as streptococci and staphylococci. Recently, angiopoietin-1, 2 and ephrin B2 agents in other vascular tumours such as Bartonellahenselae, B. quintana, and human herpes virus 8 have been postulated to play a part in recurrent pyogenic granuloma. Multiple pyogenic granulomas with satellite lesions may occur as a complication of tumour removal or trauma. Viral oncogenes, hormonal influences, microscopic arteriovenous malformation along with inclusion bodies and gene depression in fibroblasts have all been associated.

Differential diagnosis of pyogenic granuloma comprises haemangioma, peripheral giant cell granuloma, peripheral ossifying fibroma and metastatic carcinoma, and amelanotic melanoma. As the pyogenic granuloma does not spontaneously involute, it should be surgically excised with 2-mm margins at its clinical boundary and to a depth to the periosteum or to the causative agent. Any foreign body, calculus, or defective restoration should be eliminated as part of the excision. Recurrence is infrequent in non-pregnant individuals unless and until incomplete excision or failure to remove etiologic factors or re-injury to the area. ${ }^{6-10}$

Pyogenic granuloma will recur if excised during pregnancy, and it is generally accepted that treatment should be observant unless it becomes troublesome or there is a suspicion of malignancy. ${ }^{8-10}$ Even though the conventional treatment for pyogenic granuloma is surgical excision, a recurrence rate of $16 \%$ has been reported. There are also reports of the lesion being removed with electric scalpel or cryosurgery. Other approaches used by various workers include cauterization with silver nitrate, sclerotherapy with sodium tetra decylsulfate and mono-ethanolamineoleate ligation, absolute ethanol injection dye, $\mathrm{Nd}$ : YAG and $\mathrm{CO}_{2}$ laser, shave excision, and laser photocoagulation." $10-12$ Treatment part should be done carefully. Control of bleeding 
by compression of the lesion. Blood tranfusions can be done in case of severe bleeding from a pregnancy tumor. ${ }^{13,14}$ Thorough follow-up is needed because of its high recurrence rate. $^{15}$

\section{Conclusion}

Pyogenic granuloma is benign, so adequate excision should cure the lesion. Excision is the treatment of choice, but old age and recurrent or multiple lesions might be an indication for cryosurgery. Recurrent lesions could be a result of incomplete excision or treating pyogenic granuloma by cautery or laser.

\section{Clinical Significance}

"The clinical features of pyogenic granulomas of the oral cavity, although are helpful in making a differential diagnosis but are inadequate for a definite diagnosis in a given case. Further, their clinical resemblance to other inflammatory tumors, as well as to some true neoplasms of the oral cavity, necessitate an appropriate microscopic examination of the biopsy specimens, before the final diagnosis is made and adequate treatment is instituted."

\section{Source of Funding}

None.

\section{Conflict of Interest}

None.

\section{References}

1. Kerr DA. Granuloma pyogenicum. Oral Surg. 1951;4:158-76.

2. Angelopoulus AP. Pyogenic granuloma of the oral cavity: Statistical analysis of its clinical features. J Oral Surg. 1971;29:840-7.
3. Sapp JP, Eversole LR, Wysocki GP. Contemporary oral and maxillofacial pathology. 2nd ed. St. Louis, Missouri: Mosby; 2004.

4. Poncet A, Dor L. Botryomycosehumaine. Rev Chir Orthop. 1897;18:996.

5. Saravana GHL. Oral pyogenic granuloma: a review of 137cases. Br J Oral Maxillofac Surg. 2009;47:318-319.

6. Marx RE, Stern D. Oral and Maxillofacial Pathology: A rationale for diagnosis and treatment. 1st ed. Illinois: Quintessence Publishing; 2003.

7. Pyogenic granuloma on the upper lip: an unusual location. $J$ Appl Oral Sci. 2010;18:538-e41.

8. Fenton JE, Timon CI, McShane DP. Lingual granuloma gravidarum. Otolaryngol Head Neck Surg. 1996;114:682-e3.

9. Sheth SN, Gomez C, Josephson GD. Pathological case of the month. Diagnosis and discussion: pyogenic granuloma of the tongue. Arch Pediatr Adolesc Med. 2001;155:1065-e6.

10. Akyol MU, Yalçiner EG, Dogan AI. Pyogenic granuloma (lobular capillary hemangioma) of the tongue. Int J Pediatr Otorhinolaryngol. 2001;58:239-e41.

11. Shafer WC, Hine MK, Levy BM. A textbook of oral pathology 4th ed. Philadelphia: W.B. Sanders and Company; 1983;35960 .

12. Shuhert MM, Peterson DE, Lloid ME. Oralcomplications. Hematopoietic Cell Transplantation, Blackwell Science Chapter; 1998;64 751-63.

13. Kanza NK, Hutt MSR. Pyogenic granuloma: A study of 181 cases from Malawi. East Afr Med J.1981;58:319-23.

14. Powell JL, Bailey CL, Coopland AT. Nd-YAG laser excision of a giant gingival pyogenic granuloma of pregnancy. Lasers Surg Med. 1994;14:178-83.

15. Vilmann A. Pyogenic granuloma: evaluation of oral conditions. Br J Oral Maxillofac Surg. 1986;24:316-82.

How to cite this article: Ravishankar PL, Vijayan V, Rao S, Saravanan AV. Midline pyogenic granuloma of the tongue: A rare case report. Int J Oral Health Dent 2020;6(1):55-7. 